\title{
Business-managed IT - Rahmenbedingungen für mehr IT-Verantwortung durch den Fachbereich
}

\author{
Iris Hausladen · Philipp Sylla
}

Eingegangen: 4. Juni 2020 / Angenommen: 11. Juli 2020 / Online publiziert: 30. Juli 2020

(C) Der/die Autor(en) 2020

Zusammenfassung In einer zunehmend digitalen Arbeitswelt gewinnt die Frage an Bedeutung, wie IT-Aufgaben bestmöglich abgewickelt und organisiert werden können. Aktuelle Entwicklungen führen hierbei vermehrt dazu, dass IT-Tätigkeiten, mit der Zustimmung der IT-Abteilung, durch einzelne Fachbereiche übernommen werden. Im Hinblick auf diese sogenannte Business-managed IT stehen Unternehmen allerdings vor der Herausforderung, zu beurteilen, wann mehr Verantwortung von der IT-Abteilung an den Fachbereich übertragen werden kann. Durch eine systematische Literaturanalyse werden in diesem Beitrag sieben relevante Kontextfaktoren identifiziert, welche Unternehmen bei diesen Entscheidungen unterstützen sollen. Diese Faktoren werden in drei Dimensionen zusammengefasst: (1) Aufgabe (Spezifität der Applikation, Unsicherheit, Nutzungsumfang und Risiken der Applikation), (2) Mensch (IT-Wissen im Fachbereich und Fachbereichswissen in der IT-Abteilung) und (3) Technologie (Grad der Applikationsintegration).

Schlüsselwörter Business-managed IT $\cdot$ Schatten-IT $\cdot$ IT-Governance $\cdot$ Situativer Ansatz

I. Hausladen · P. Sylla $(\square)$

Heinz Nixdorf-Lehrstuhl für IT-gestützte Logistik, HHL Leipzig Graduate School of Management, Jahnallee 59, 04109 Leipzig, Deutschland

E-Mail: philipp.sylla@hhl.de

I. Hausladen

E-Mail: iris.hausladen@hhl.de 


\title{
Business-managed IT —Conditions Fostering Responsibilities for IT Within Business Units
}

\begin{abstract}
In an increasingly digital work environment, the question of how IT tasks can be handled and organized in the best possible way is becoming increasingly important. Current trends lead to more and more IT tasks being taken over by business units with the approval of the IT department. With regard to this so-called Business-managed IT, however, companies are faced with the challenge of assessing when more responsibility can be transferred from the IT department to the business employees. This paper uses a systematic literature analysis to identify relevant contingency factors which can support companies in these decisions. The identified factors are structured along three dimensions: (1) task (specificity, uncertainty, scope of use and risks), (2) people (IT-knowledge in the business department and business-knowledge in the IT department) and (3) technology (degree of application integration).
\end{abstract}

Keywords Business-managed IT $\cdot$ Shadow IT $\cdot$ IT Governance $\cdot$ Contingency Theory

\section{Motivation und Zielsetzung}

Die Digitalisierung eröffnet vielfältige Chancen, bewirkt jedoch auch einen tiefgreifenden Wandel in vielen Unternehmensbereichen (Hausladen 2016, S. 17). Dies betrifft auch die Organisation der IT-Aufgaben zwischen dem Fachbereich und der IT-Abteilung. Hierbei sind seit einiger Zeit Trends zu beobachten, die zu einer Verschiebung von Verantwortlichkeiten für IT-Aufgaben von der IT-Abteilung hin zum Fachbereich (Business) führen. Sogenannte Digital Natives sind vermehrt im Arbeitsleben aktiv. Diese sind nach 1980 geboren und mit sozialen Medien, dem Internet und einfach zu bedienenden Websites aufgewachsen. Es kann davon ausgegangen werden, dass diese im Vergleich zu vorhergehenden Generationen andere Fähigkeiten und Ansprüche an die IT haben werden (Palfrey und Gasser 2008). Weiterhin können durch eine steigende Verbreitung des Cloud Computings viele ITDienste über das Internet bezogen werden, ohne das diese in unternehmensinternen Rechen- und Datenzentren betrieben werden müssen. Dies bietet dem Fachbereich die Möglichkeit, Applikationen in der Cloud (bspw. ein CRM-System) im Zweifel auch ohne eine Unterstützung durch die IT-Abteilung zu verwenden. Außerdem werden private Endgeräte und Applikationen zunehmend am Arbeitsplatz eingesetzt - ein Trend, der als IT-Konsumerisierung bezeichnet wird (Harris et al. 2012). Vor diesem Hintergrund postulieren einige Autoren, dass sich die IT-Governance in einer Art und Weise verändern muss, die den Fachbereichen mehr Verantwortung für IT-Aufgaben überträgt (Andriole 2015).

In diesem Zusammenhang ist der Begriff Business-managed IT in die Diskussion eingeführt worden (Kopper et al. 2018). Dieser bezeichnet die offen kommunizierte und mit der IT-Abteilung abgestimmte Erstellung, den Einkauf oder das Management von Software, Hardware oder IT-Serviceprozessen durch den Fachbereich. 
Für Unternehmen kann Business-managed IT einige Vorteile bieten, darunter eine Reduzierung der Arbeitsbelastung häufig unterbesetzter IT-Abteilungen und die Erhöhung von Agilität sowie Autonomie des Fachbereichs.

Neben diesen Vorteilen stellt die Business-managed IT die Unternehmen jedoch vor neue Herausforderungen. Unternehmen sind mit vielfachen Anfragen zur Einführung neuer Applikationen konfrontiert. Für die umzusetzenden Anwendungen stehen sie hierbei vor der Aufgabe, die Verantwortlichkeiten hinsichtlich der ITAufgaben zwischen dem Fachbereich und der IT-Abteilung festzulegen. Dabei muss ein hoher Anteil an Verantwortung für IT-Aufgaben im Fachbereich bzw. Businessmanaged IT nicht für alle Applikationen die richtige Entscheidung sein. So liegen die Vorteile einer zentralen Veranwortung der IT-Abteilung unter anderem in der Ausnutzung von Synergien und den generierten Vorteilen durch Standardisierung (Peterson 2004). Es stellt sich daher die Frage, unter welchen Bedingungen ein mehr an Verantwortung für IT-Aufgaben im Fachbereich für Unternehmen sinnvoll ist. Bislang bietet die Literatur zu dieser konkreten Problemstellung jedoch noch keine Hilfestellung.

Das Forschungsziel dieses Beitrags ist es daher zu untersuchen, unter welchen Bedingungen ein hoher Anteil der IT-Aufgaben in der Verantwortung des Fachbereichs liegen kann und somit Business-managed IT sinnvoll ist. Im Vordergrund soll hierbei die Identifikation von Kontextvariablen liegen, welche die Entscheidung zur Verteilung von Verantwortlichkeiten zwischen der IT-Abteilung und dem Fachbereich beeinflussen. Unter Kontextvariablen sind diejenigen Größen zu verstehen, „[...] bei denen begründet davon ausgegangen werden kann, dass sie den in der Untersuchung erforschten Gestaltungsbereich (1) beeinflussen oder (2) zumindest von den agierenden Personen bei der Entscheidung über die Ausprägung der Gestaltungsvariablen zu berücksichtigen sind“ (Wolf 2011, S. 38). Im Rahmen dieses Beitrags sind also Variablen gemeint, die von Entscheidern bei der Verteilung der Verantwortlichkeiten für IT-Aufgaben berücksichtigt werden sollten.

Zur Beantwortung dieser Forschungsfrage wurde eine Literaturanalyse durchgeführt, welche im nächsten Abschnitt beschrieben wird. Im Anschluss werden die Implikationen für das IT-Management diskutiert. Schließlich bildet eine kurze Zusammenfassung den Abschluss dieses Beitrags.

\section{Literaturanalyse}

Das Konzept der Business-managed IT ist aus der Literatur entstanden, die sich mit der sogenannten Schatten-IT beschäftigt. Im Gegensatz zur Business-managed IT werden bei der Schatten-IT Aufgaben in Bezug auf IT von den Fachbereichen bzw. Nutzern übernommen, ohne dass die unternehmenseigene IT-Abteilung einbezogen wird (Kopper et al. 2017). Der Unterschied zwischen diesen beiden Konzepten ist folglich die Zustimmung bzw. der Einbezug der IT-Abteilung in IT-Implementierungen, die vom Fachbereich umgesetzt werden. Gemeinsam ist beiden Konzepten jedoch, dass die Fachbereiche Verantwortung für IT-Aufgaben übernehmen. Aus diesem Grund sowie der Tatsache, dass es seit geraumer Zeit Forschung zum Konzept der Schatten-IT und bislang nur wenig Erkenntnisse zum Gebiet der Business- 
managed IT gibt, wurde die Literatur zur Schatten-IT als Basis für die Literaturanalyse gewählt.

Als forschungsleitende Grundlage der Literaturanalyse wurde der situative Ansatz gewählt. Gemäß dieses Ansatzes entsteht Erfolg unter der Bedingung, dass es einen Zusammenhang zwischen den Kontextbedingungen und den Gestaltungsmaßnahmen gibt. In Bezug auf die vorliegende Untersuchung stellt die Verteilung der Verantwortlichkeiten zwischen IT-Abteilung und Fachbereich die relevante Gestaltungsmaßnahme dar. Diese Verteilung der Verantworlichkeiten wird derart verstanden, dass sie sich auf einem Spektrum zwischen reiner Verantwortung durch die ITAbteilung oder durch den Fachbereich bewegen kann. Hierbei wird angenommen, dass es eine Bandbreite an Mischformen zwischen diesen Extrempolen geben kann. Ziel des Beitrags ist die Identifikation von Kontextvariablen, welche tendenziell zur einer Verschiebung der Verantwortlichkeiten in Richtung einer der beiden polaren Enden dieses Spektrums führen.

\subsection{Vorgehen}

Die Literaturrecherche erfolgte in drei Schritten. Zuerst wurde eine Suche nach geeigneter Literatur in den folgenden Datenbanken durchgeführt: AIS Electronic Library, Business Source Complete (EBSCO) und SpringerLink. Für die Literatursuche wurden verschiedene Schlüsselwörter kombiniert. Zum einen wurden die Wörter „Schatten“, ,,shadow“ und ,feral“ mit „IT“, ,System“ bzw. ,systems“ und „Projekt“ bzw. ,projects“ kombiniert. Zum anderen wurde nach den Begriffen „Business-managed IT“ und ,end-user development“ gesucht. Durch dieses Vorgehen konnten 494 Artikel identifiziert werden. In einem zweiten Schritt wurden im Anschluss die Artikel hinsichtlich Informationen in Titel, Abstract und Schlüsselwörtern untersucht, wodurch die Menge der Artikel auf 91 Artikel reduziert werden konnte. In einem dritten Schritt wurden diese Artikel genauer untersucht und gegebenenfalls Vorwärts- und Rückwärtssuchen durchgeführt. Hierbei wurden 21 Artikel identifiziert, die Hinweise über potentiell relevante Kontextvariablen liefern.

\subsection{Ergebnis: Identifizierte Kontextvariablen}

Die 21 identifizierten, relevanten Artikel wurden wiederholt gelesen und einschlägige Textpassagen wurden markiert und kodiert. Durch dieses iterative Vorgehen wurden insgesamt sieben Kontextvariablen identifiziert: Spezifität der Applikation, Unsicherheit, Nutzungsumfang der Applikation, Risiken der Applikation, IT-Wissen im Fachbereich, Fachbereichswissen in der IT-Abteilung und der Grad der Applikationsintegration. Diese werden nun im Folgenden diskutiert, wobei IT-Wissen im Fachbereich und Fachbereichswissen in der IT-Abteilung aufgrund der thematischen Ähnlichkeit gemeinsam in einem Abschnitt vorgestellt werden.

Die ersten beiden Kontextvariablen haben dabei einen starken Bezug zur Transaktionskostentheorie. Diese geht davon aus, dass die Eignung verschiedener Organisationsformen durch die Transaktionskosten bestimmt wird, welche wiederum von Merkmalen der Transaktion abhängig sind. In der Unternehmenspraxis sollten sich dabei diejenigen Organisationsformen durchsetzen, die in dem jeweiligen 
Einsatzgebiet am effizientesten sind und somit die geringsten Transaktionskosten aufweisen.

\subsubsection{Spezifität der Applikation}

Die Spezifität der Applikation kann als Funktionsspezifität verstanden werden, die sich auf die Anpassung der Applikation an die spezifischen Anforderungen des Fachbereichs bezieht. Im Sinne der Transaktionskostentheorie erhöhen sich mit steigender Spezifität der Applikation die Aufwände zur Kommunikation, die zwischen Fachbereich und IT-Abteilung zur Implementierung und zum Betrieb einer Applikation notwendig sind. Daher sollte eine höhere Spezifität der Applikation auch mit mehr Verantwortung des Fachbereichs einhergehen (Winkler et al. 2011; Winkler und Benlian 2012; Winkler und Brown 2013; Zimmermann et al. 2016). Als weitere Begründung hierfür kann angeführt werden, dass für spezifische IT-Systeme auch spezifisches Wissen, beispielsweise hinsichtlich der zu unterstützenden Geschäftsprozesse, benötigt wird. Da dieses normalerweise verstärkt in dem betroffenen Fachbereich und nicht in der IT-Abteilung vorliegt, ist die Übernahme von Aufgaben durch die IT-Abteilung weniger vorteilhaft (Fürstenau et al. 2017; Kopper et al. 2018; Zimmermann 2018).

Die empirischen Erkenntnisse hinsichtlich der Bedeutung der Spezifität der Applikation sind bislang noch nicht eindeutig. Einige Fallstudien-Untersuchungen bieten Hinweise für die Relevanz dieser Kontextvariable. Kopper et al. (2018) berichten von einem IT-System, welches in einem Produktionswerk eines Maschinenbauunternehmens eingesetzt wird. Da das IT-System spezifische Geschäftsprozesse des Werks unterstützt, liegt die Verantwortung größtenteils in der Verantwortung des Fachbereichs. Die IT-Abteilung ist lediglich für die Bereitstellung der IT-Infrastruktur und die Schnittstellenbetreuung zuständig. Ähnliche Hinweise finden sich auch in weiteren qualitativen Untersuchungen (Fürstenau et al. 2017; Zimmermann und Rentrop 2014; Zimmermann et al. 2016). In den wenigen quantitativen Untersuchungen, in denen die Auswirkung der Spezifität der Applikation auf die Verteilung der Verantwortung für IT-Aufgaben zwischen IT-Abteilung und Fachbereich bislang untersucht wurde, konnte jedoch kein statistisch signifikanter Einfluss der Spezifität nachgewiesen werden (Winkler und Benlian 2012; Winkler und Brown 2013).

\subsubsection{Unsicherheit}

Wie die Spezifizität der Applikation kann auch die Wirkung der Unsicherheit mit Hilfe der Transaktionskostentheorie begründet werden. Hierbei wird die Unsicherheit der Umwelt derart verstanden, dass beispielsweise neue Technologien oder deren Nachfrage nur schwer vorhersehbar sind. Bei steigender Spezifizität erhöht die Unsicherheit die Transaktionskosten, da die ungewissen zukünftigen Entwicklungen unter anderem die Verhandlungen und den Vertragsabschluss schwieriger und komplexer gestalten können. Im speziellen Fall der Einführung von IT-Applikationen kann sich die Unsicherheit beispielsweise als Bedarfsunsicherheit, Unsicherheit hinsichtlich IT-Anforderungen oder bezüglich künftiger Änderungen niederschlagen (Zimmermann 2018, S. 60). Die Aufgabenverteilung als auch die Abstimmung 
zwischen IT-Abteilung und Fachbereich wird mit zunehmender Unsicherheit rigide und ineffizient. Es kann daher davon ausgegangen werden, dass mit steigender Unsicherheit mehr Verantwortung für IT-Aufgaben im Fachbereich übernommen wird.

In der Literatur lassen sich bereits einige Hinweise auf die Bedeutung dieser Kontextvariable finden. Bygstad (2017) unterscheidet zwischen schwergewichtigen ITSystemen, die von der IT-Abteilung systematisch entworfen werden, und leichtgewichtigen IT-Systemen, die von Nutzern und externen Anbietern entwickelt werden. Während die Entwicklungskultur der IT-Abteilung hinsichtlich schwergewichtiger IT-Systeme von Systematik, Qualitäts- und Sicherheitsdenken geprägt ist, geht es dem Fachbereich im Rahmen von leichtgewichtigen IT-Systemen um Innovation und das Experimentieren. Auch in einigen empirischen Untersuchungen, die auf Fallstudien basieren, konnten Hinweise für die Bedeutung der Unsicherheit für die Übernahme von Verantwortung durch den Fachbereich gefunden werden. Györy et al. (2012) identifizieren Schatten-IT als einen Haupttreiber für IT-Innovationen. Kopper et al. (2018) beschreiben den Fall eines Handelsunternehmens, welches sich von einem Kataloghändler zu einem E-Commerce Unternehmen entwickelt hat. Da das Unternehmen in einer turbulenten Umwelt mit schwierig vorherzusagenden zukünftigen Marktentwicklungen operiert, sind die Anforderungen an die IT-Systemlandschaft unsicher. Daher wurde das Webshop-System mit Zustimmung der ITAbteilung sowie der Geschäftsführung durch den Fachbereich entwickelt. Ähnliche Hinweise lassen sich auch in den Untersuchungen von Zimmermann et al. (2016) und Zimmermann et al. (2017) finden.

\subsubsection{Nutzungsumfang der Applikation}

Der Nutzungsumfang der Applikation bezeichnet die Breite, in der eine Anwendung innerhalb des Unternehmens verwendet wird (Winkler und Brown 2013). So können beispielsweise Anwendungen in der Funktionalität auf bestimmte Unternehmensfunktionen (wie Personalwesen oder Vertrieb) beschränkt oder von vielen Unternehmensfunktionen genutzt werden (z. B. Kommunikationsapplikationen). Eine Möglichkeit zur Abstufung des Nutzungsumfangs bietet die Unterscheidung nach abteilungsspezifischen, abteilungsübergreifenden, unternehmensweiten und interorganisationalen IT-Systemen (Xue et al. 2008). Visuell kann die Breite des Anwendungsbreichs mit der Hilfe von sogenannten IT-Bebauungsplänen dargestellt werden. Zum Beispiel könnte man somit aufzeigen, über welche Prozesse und welche Sparten innerhalb eines Unternehmens eine Applikation Anwendung findet (Durst 2008). Weiterhin kann die Anzahl der Nutzer des IT-Systems als Dimension des Nutzungsumfangs verstanden werden (Rentrop und Zimmermann 2012).

Ein höherer Nutzungsumfang der Applikation sollte hierbei mit mehr Verantwortung für IT-Aufgaben durch die IT-Abteilung einhergehen. IT-Systeme, die Prozesse über organisationale Grenzen (wie Abteilungen oder Unternehmen) hinweg unterstützen, erfordern mehr Ressourcen und haben größere Auswirkungen auf das Unternehmen (Xue et al. 2008). Daher ist es aufgrund von Kosten- und Risikoüberlgungen in Fällen eines hohen Nutzungsumfangs sinnvoller, die IT-Abteilung mit einzubeziehen (Zimmermann et al. 2016). Während Entscheidungen und Aufga- 
ben für abteilungsspezifische Applikationen meist direkt von Mitarbeitern aus dem Fachbereich übernommen werden können, erhöht die Anzahl der involvierten Abteilungen und Nutzer die Komplexität und das Ausmaß der Nutzeranfragen. In diesen Fällen ist eine zentrale Übernahme bestimmter Tätigkeiten durch die IT-Abteilung angebracht (Winkler et al. 2011).

Die bisherigen empirischen Erkenntnisse bieten einige Hinweise für die Bedeutung dieser Kontextvariable. In mehreren qualitativen Untersuchungen wurde von dem Einfluss des Nutzungsumfangs auf die Verteilung der Verantwortung zwischen IT-Abteilung und Fachbereich berichtet (Chua et al. 2014; Zimmermann et al. 2016, 2017). Zudem konnte in zwei quantitativen Untersuchungen ein Einfluss des Nutzungsumfangs der Applikation auf die Verteilung der Verantwortlichkeiten für ITAufgaben und Entscheidungen nachgewiesen werden (Winkler und Benlian 2012; Winkler und Brown 2013).

\subsubsection{Risiken der Applikation}

Risiken von Applikationen können verschiedene Ursachen haben. Beispielsweise kann die Verfügbarkeit von Applikationen gefährdet sein. Gezielte Angriffe oder technische Ausfälle können zu Ausfallzeiten von Applikationen führen. In diesem Fall haben die Nutzer nicht mehr die Möglichkeit, die darin enthaltenen Dienste und Daten der Applikation zu nutzen. Das Ausmaß der Folgen wird jedoch nicht für alle Applikationen identisch sein. So kann das Spektrum von geringfügigen bis hin zu schweren Schäden reichen. Ein weiterer wichtiger Aspekt ist sicherlich die Sensibilität der Daten, die in der Applikation verarbeitet werden. Bestimmte Daten bringen Datenschutzprobleme mit sich, beispielsweise wenn diese zur Identifizierung einer Person verwendet werden können und es sich um sensible Informationen (z.B. Religion) handelt. Das Ausmaß der sensiblen Daten, welche in Applikationen verarbeitet wird, kann jedoch sehr unterschiedlich ausfallen.

Wenn eine Applikation höhere Risiken aufweist, so sollte der Anteil des Einbezugs der IT-Abteilung höher ausfallen. Zum einen besteht die Gefahr, dass den Fachbereichen kritische Elemente eines sicheren IT-Betriebs nicht bekannt sind (Chua und Storey 2016). Zum anderen kann der Einbezug der IT-Abteilung potentielle Risiken reduzieren. Durch die zentrale Steuerung der Systeme durch die IT-Abteilung wird das Setzen von Standards ermöglicht. Dies führt zu einer verbesserten Interoperabilität der Systeme und geringeren technischen Risiken, da genehmigte Systemkomponenten in der Regel weniger Kompatibilitätsprobleme aufweisen. Weiterhin sind die Mitarbeiter der zentralen IT-Abteilungen in der Regel hochqualifiziert. Der Einsatz von strukturierten Systementwurfs- und Wartungsverfahren durch diese Mitarbeiter soll zu zuverlässigen IT-Systemen führen (Warkentin und Johnston 2008).

Es gibt bereits einige empirische Erkenntnisse, die Indikationen für die beschriebene Wirkung bieten. Zimmermann et al. (2017) finden in mehreren Fallstudien Hinweise dafür, dass es den oben beschrieben positiven Zusammenhang zwischen den Risiken der Applikation und Verantwortung der IT-Abteilung gibt. Ähnliche Hinweise liefern auch weitere qualitative Studien (Chua et al. 2014; Fürstenau et al. 2017; Zimmermann et al. 2016). Allerdings gibt es auch einige weitere Untersuchun- 
gen, die den postulierten Zusammenhang nicht bestätigen können. So finden Rentrop et al. (2015), dass etwa 55\% der untersuchten Schatten-IT Systeme prozessrelevant und somit, aus einer Risikoperspektive, als kritisch anzusehen sind. Weiterhin kann Haag (2015) in einer quantitativen Untersuchung keinen statistisch signifikanten Zusammenhang zwischen den wahrgenommenen Sicherheitsrisiken und der Intention der Verwendung von Schatten-IT finden.

\subsubsection{IT-Wissen im Fachbereich und Fachbereichswissen in der IT-Abteilung}

Das IT-Wissen im Fachbereich bezieht sich darauf, wie gut der Einzelne grundlegende IT-Konzepte versteht und wie gut dieser über die IT in seinem Unternehmen informiert ist. IT-Wissen ist ein wichtiger Faktor, um die Führungsrolle von Managern beim Einsatz von IT in Unternehmen zu erklären (Bassellier et al. 2003). Außerdem ist ein ausreichendes Verständnis für IT und IT-Managementpraktiken eine wichtige Voraussetzung für eine dezentrale Ausgestaltung von IT (Sambamurthy und Zmud 1999). Daher kann von einem positiven Zusammenhang zwischen IT-Wissen im Fachbereich und der Übernahme von Verantwortung für IT-Aufgaben durch diesen ausgegangen werden. Spiegelbildlich sollte ein hohes Wissen über Fachbereichsthemen in der IT-Abteilung, beispielsweise Wissen über die Geschäftsprozesse, einen positiven Zusammenhang zur Übernahme von Verantwortung durch die IT-Abteilung haben (Winkler und Brown 2013).

Bislang gibt es nur sehr wenige Erkenntnisse in der Literatur, die für die Auswirkungen auf Business-managed IT relevant sind. Kopper und Westner (2016) identifizieren eine hohe IT-Kompetenz von Nutzern als einen Faktor, der die Entstehung von Schatten-IT positiv beeinflusst. Winkler und Brown (2013) finden in einer quantitativen Untersuchung einen positiven Zusammenhang zwischen dem Fachbereichswissen in der IT-Abteilung und dem Grad der Verantwortung für IT-Aufgaben durch diese. Es konnte jedoch kein Einfluss des IT-Wissens im Fachbereich nachgewiesen werden.

\subsubsection{Grad der Applikationsintegration}

Der Grad der Applikationsintegration bezeichnet das Ausmaß, in dem eine Applikation mit anderen Applikationen verbunden ist. Eine Möglichkeit, den Grad der Applikationsintegration messbar zu machen, könnte beispielsweise darin bestehen, die Anzahl der Schnittstellen zu anderen Applikationen zu beziffern.

Mehrere Gründe sprechen für einen positiven Zusammenhang zwischen dem Grad der Applikationsintegration und der Übernahme von Verantwortung in der IT-Abteilung. Erstens stellt die Entwicklung technischer Schnittstellen die Geschäftsanwender vor Herausforderungen. Hetzenecker et al. (2012) kommen zu dem Schluss, dass die IT-Abteilung für Integrationsfragen weiterhin benötigt wird. Sie begründen diese Aussage mit der Vermutung, dass (1) die Fachbereiche nur wenig über die bestehende IT-Infrastruktur wissen und über mangelnde konzeptionelle Fähigkeiten zum Aufbau einer Enterprise IT-Architektur verfügen, (2) ein tiefes Verständnis und Wissen über verschiedene Implementierungsmöglichkeiten im Zusammenhang mit weniger standardisierten Schnittstellen erforderlich ist und (3) die Endanwender da- 
zu neigen, Insellösungen zur Lösung aktueller Probleme zu schaffen, während ein ganzheitlicher Ansatz zur IT-Architektur erforderlich ist. Weiterhin spricht für einen Einbezug der IT-Abteilung für Applikationen mit hohem Integrationsgrad das erhöhte Risiko von Systemausfällen. Wenn Applikationen hochgradig integriert sind, spielen sie eine zentrale Rolle in einem Netzwerk von Anwendungen. Dies führt jedoch zu größeren Schäden wenn eine solche zentrale Applikationen nicht verfügbar ist, da andere Applikationen von dieser abhängig sein können (Fürstenau und Rothe 2014). Aus denselben Gründen, die bereits hinsichtlich der Risiken der Applikation diskutiert wurden, sollten daher mehr Aufgaben in der Verantwortung der ITAbteilung liegen.

Auch für den Grad der Applikationsintegration gibt es bislang nur sehr wenige empirische Erkenntnisse. Hetzenecker et al. (2012) beobachten im Rahmen einer Fallstudie in einem Hotel, dass der Fachbereich für die Ausgestaltung von Schnittstellen zwischen Applikationen noch immer von der IT-Abteilung abhängig ist. Winkler et al. (2011) beobachten in vier Fallstudien unterschiedliche Grade der Integrationskomplexität von Applikationen. Sie kommen jedoch zumindest hinsichtlich eines der Fallbeispiele zu dem Schluss, dass der Einfluss dieser Kontextvariable auf die Verteilung der Veranwortlichkeiten zwischen Fachbereich und IT-Abteilung nicht stark ist.

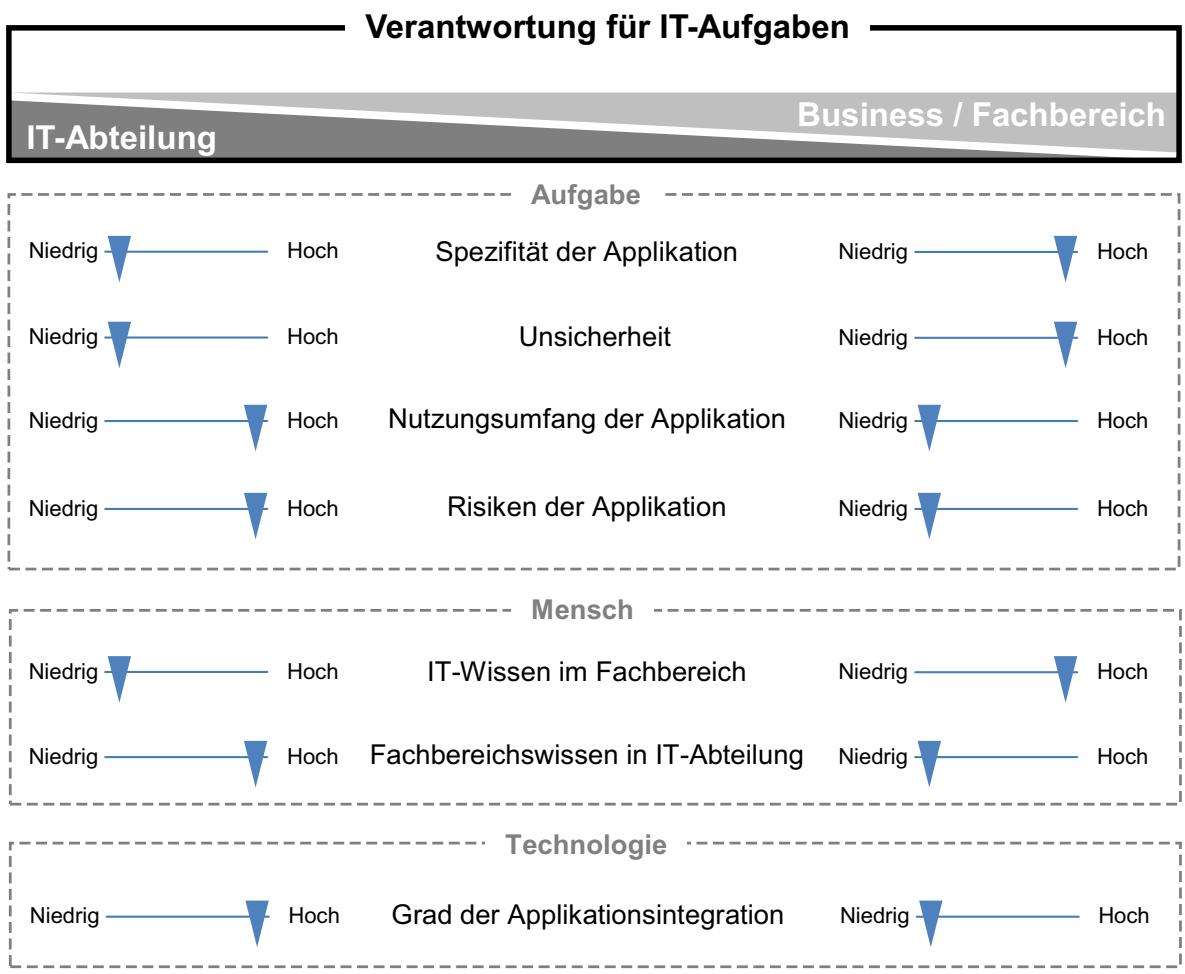

Abb. 1 Relevante Kontextvariablen zur Entscheidungsunterstützung 


\section{Implikationen für das IT-Management}

Verantwortliche aus dem Bereich des IT-Management müssen vermehrt entscheiden, unter welchen Bedingungen Business-managed IT sinnvoll ist. Abb. 1 gibt einen Überblick über die identifizierten Kontextvariablen, welche diese Entscheidung unterstützen können.

Die sieben identifizierten Kontextvariablen wurden hierbei in drei übergeordneten Dimensionen zusammengefasst: Aufgabe, Mensch und Technologie. Diese Dimensionen haben ihren Ursprung im Leavitt-Diamanten für organisationalen Wandel. Das Modell von Leavitt ist in diesem Zusammenhang relevant, da die Einführung von Applikationen mit technologischen und sozialen Veränderungen verbunden ist. Leavitt schlägt vor, dass vier voneinander abhängige Variablen für solche Veränderungen berücksichtigt werden müssen: Aufgabe, Menschen, Technologie und Struktur (Leavitt 1965). In diesem Beitrag wird die Dimension der Struktur als die Verteilung der Verantwortlichkeiten zwischen IT-Abteilung und Fachbereich, in Abhängigkeit der drei Dimensionen Aufgabe, Mensch und Technologie betrachtet. Die Dimensionen und die darin enthaltenen Kontextvariablen können für Entscheidungen zur Verteilung von Verantwortlichkeiten für IT-Aufgaben eine erste Orientierungshilfe bieten.

\section{Zusammenfassung und Ausblick}

Dieser Beitrag sollte ergründen, unter welchen Bedingungen ein hoher Anteil der ITAufgaben in der Verantwortung des Fachbereichs liegen kann und somit Businessmanaged IT tendenziell sinnvoll ist. Durch eine systematische Literaturanalyse konnten sieben relevante Kontextvariablen identifiziert werden, welche bei Entscheidungen hinsichtlich der Verteilung der Verantwortung für IT-Aufgaben beachtet werden sollten.

Zusammengefasst zu drei übergeordneten Dimensionen können diese Kontextvariablen den verantwortlichen Entscheidern im IT-Management eine Unterstützung bieten, wann ein höherer Anteil an Verantwortung für IT-Aufgaben im Fachbereich sinnvoll ist. Mitunter sollten hierbei menschliche, technologische und aufgabenspezifische Aspekte der Applikationen beachtet werden.

In vielerlei Hinsicht besteht jedoch für die in diesem Beitrag betrachtete Problemstellung weiterer Forschungsbedarf. So zeigt sich, dass mit dem Grad der Applikationsintegration bislang nur eine Kontextvariable für die technologische Dimension identifiziert wurde. Es ist jedoch davon auszugehen, dass auch weitere Aspekte der Technologie als Treiber für mehr IT-Verantwortung im Fachbereich relevant sind. Daher sollten zukünftige Forschungsvorhaben besonders die technologischen Rahmenbedingungen beleuchten. Interessant ist hierbei beispielsweise, inwiefern Cloud Computing als ein Treiber für Business-managed fungiert. Weiterhin ist bislang wenig darüber bekannt, in welchen Formen eine Verteilung der Verantwortlichkeiten zwischen IT-Abteilung und Fachbereich ausgeprägt sein kann. Weitere Forschungsbemühungen könnten daher zur Erarbeitung von möglichen Strukturierungen (beispielsweise anhand von Prozessmodellen oder Typologisierungen) dieses Spektrums 
zwischen der kompletten Verantwortung für IT-Aufgaben im Fachbereich oder in der IT-Abteilung vorgenommen werden.

Funding Open Access funding provided by Projekt DEAL.

Open Access Dieser Artikel wird unter der Creative Commons Namensnennung 4.0 International Lizenz veröffentlicht, welche die Nutzung, Vervielfältigung, Bearbeitung, Verbreitung und Wiedergabe in jeglichem Medium und Format erlaubt, sofern Sie den/die ursprünglichen Autor(en) und die Quelle ordnungsgemäß nennen, einen Link zur Creative Commons Lizenz beifügen und angeben, ob Änderungen vorgenommen wurden.

Die in diesem Artikel enthaltenen Bilder und sonstiges Drittmaterial unterliegen ebenfalls der genannten Creative Commons Lizenz, sofern sich aus der Abbildungslegende nichts anderes ergibt. Sofern das betreffende Material nicht unter der genannten Creative Commons Lizenz steht und die betreffende Handlung nicht nach gesetzlichen Vorschriften erlaubt ist, ist für die oben aufgeführten Weiterverwendungen des Materials die Einwilligung des jeweiligen Rechteinhabers einzuholen.

Weitere Details zur Lizenz entnehmen Sie bitte der Lizenzinformation auf http://creativecommons.org/ licenses/by/4.0/deed.de.

\section{Literatur}

Andriole SJ (2015) Who owns IT? Commun ACM 58(3):50-57. https://doi.org/10.1145/2660765

Bassellier G, Izak B, Reich BH (2003) The Influence of Business Managers' IT Competence on Championing IT. Inf Syst Res 14(4):317-336

Bygstad B (2017) Generative innovation: a comparison of lightweight and heavyweight IT. J Inf Technol 32(2):180-193

Chua CEH, Storey VC (2016) Bottom-up enterprise information systems: rethinking the roles of central IT departments. Commun ACM 60(1):66-72

Chua C, Storey V, Chen L (2014) Central IT or Shadow IT? Factors shaping users' decision to go rogue with IT. In: Proceedings of the 35th International Conference on Information Systems (ICIS) Auckland

Durst M (2008) Wertorientiertes Management von IT-Architekturen. Deutscher Universitäts-Verlag, Wiesbaden, S 38-45

Fürstenau D, Rothe H (2014) Shadow IT systems: discerning the good and the evil. In: Proceedings of the 22nd European Conference on Information Systems (ECIS) Tel-Aviv

Fürstenau D, Rothe H, Sandner M (2017) Shadow systems, risk, and shifting power relations in organizations. Commun ACM 41(1):43-61

Györy A, Cleven A, Uebernickel F, Brenner W (2012) Exploring the shadows: IT governance approaches to user-driven innovation. In: Proceedings of the 20th European Conference on Information Systems (ECIS) Barcelona

Haag S (2015) Appearance of dark clouds?-An empirical analysis of users' shadow sourcing of cloud services. In: Proceedings der Wirtschaftsinformatik Osnabrück

Harris J, Ives B, Junglas I (2012) IT consumerization: When gadgets turn into enterprise IT tools. MISQE 11(3):99-112

Hausladen I (2016) IT-gestützte Logistik. Systeme - Prozesse - Anwendungen. Springer, Wiesbaden

Hetzenecker J, Sprenger S, Kammerer S, Amberg M (2012) The unperceived boon and bane of cloud computing: end-user computing vs. Integration. In: Proceedings of the 18th Americas Conference on Information Systems (AMCIS) Seattle

Kopper A, Westner M (2016) Deriving a framework for causes, consequences, and governance of shadow it from literature. In: MKWI 2016 proceedings, S 1687-1698

Kopper A, Westner M, Strahringer S (2017) Kontrollierte Nutzung von Schatten-IT. HMD Prax Wirtschaftsinform 54(1):97-110

Kopper A, Fuerstenau D, Zimmermann S, Rentrop C, Rothe H, Strahringer S, Westner M (2018) Businessmanaged IT: a conceptual framework and empirical illustration. In: The 26th European Conference on Information Systems (ECIS) Portsmouth 
Leavitt HJ (1965) Applied organizational change in industry: structural, technological and humanistic approaches. In: March JG (Hrsg) Handbook of organizations. Rand-MacNally, Chicago, S 1144-1170

Palfrey J, Gasser U (2008) Born digital. Understanding the first generation of digital natives. Basic Books, New York

Peterson R (2004) Crafting information technology governance. Inf Syst Manag 21(4):7-22

Rentrop C, Zimmermann C (2012) Shadow IT - Management and control of unofficial IT. In: Proceedings of the Federated Conference on Computer Science and Information Systems (FedCSIS), Wrocław, Poland

Rentrop C, Zimmermann S, Huber M (2015) Schatten-IT ein unterschätztes Risiko. In: DACH Security, S 291-300

Sambamurthy V, Zmud RW (1999) Arrangements for information technology governance: a theory of multiple contingencies. MIS Q 23(2):261-290

Warkentin M, Johnston AC (2008) IT Governance and Organizational Development for Security Management. In: Straub DW, Goodman SE, Baskerville R (Hrsg) Information security, M.E. Sharpe, Armonk, S 46-68

Winkler TJ, Benlian A (2012) The dual role of IS specificity in governing software as a service. In: The 33rd International Conference on Information Systems Orlando

Winkler TJ, Brown CV (2013) Horizontal allocation of decision rights for on-premise applications and software-as-a-service. J Manag Inf Syst 30(3):13-47

Winkler TJ, Goebel C, Benlian A, Bidault F, Günther O (2011) The impact of software as a service on IS authority-a contingency perspective. In: The 32nd International Conference on Information Systems (ICIS) Shanghai

Wolf J (2011) Organisation, Management, Unternehmensführung. Theorien, Praxisbeispiele und Kritik, 4. Aufl. Gabler, Wiesbaden

Xue Y, Liang H, Boulton WR (2008) Information technology governance in information technology investment decision processes: the impact of investment characterisics, external environment, and internal context. MIS Q 32(1):67-96

Zimmermann S (2018) Der Umgang mit Schatten-IT in Unternehmen. Eine Methode zum Management intransparenter Informationstechnologie. Springer, Wiesbaden

Zimmermann S, Rentrop C (2014) On the emergence of shadow IT-A transaction cost-based approach. In: Proceedings of the 22nd European Conference on Information Systems Tel-Aviv

Zimmermann S, Rentrop C, Felden C (2016) Governing IT activities in business workgroups. Design principles for a method to control identified shadow IT. In: Witold A, Rainer A, Bogdan F (Hrsg) Business information systems 19th International Conference, BIS 2016, Leipzig, 6-8 July 2016. Lecture Notes in Business Information Processing,, Bd. 255. Springer, Cham, S 252-264

Zimmermann S, Rentrop C, Felden C (2017) A multiple case study on the nature and management of shadow information technology. J Inf Syst 31(1):79-101. https://doi.org/10.2308/isys-51579 